\title{
Payment Reform, Medication Use, and Costs: Can We Afford to Leave Out Drugs?
}

\author{
Natasha Parekh, MD, MS ${ }^{1,2}$, Mark McClellan, MD, PhD ${ }^{3}$, and William H. Shrank, MD, MSHS \\ 'Division of General Internal Medicine, University of Pittsburgh, Pittsburgh, PA, USA; ${ }^{2}$ Center for High-Value Health Care and Center for Value-Based \\ Pharmacy Initiatives, UPMC Health Plan, Pittsburgh, PA, USA; ${ }^{3}$, Duke Margolis Health Center for Policy, Washington, DC, USA.
}

\begin{abstract}
Medications are one of the fastest growing sources of costs in the health system and the cornerstone of disease management. Despite extensive attention around drug pricing, medications have largely been excluded from CMSderived, value-based payment models. In this perspective, we synthesize evidence about the impact of three prominent models-primary care-based redesign, ACOs, and bundled payment programs-on medication use, adherence, and costs. We also examine the literature describing similar models implemented by private payors and their relationship with medication use and costs. The exclusion of drug costs from payment reform model design has led to missed opportunities for payors and providers to prioritize effective medication management strategies and has limited our learning about the effects on cost and quality. New CMS-based models are starting to allow greater flexibility in pharmacy benefit design and reward improved medication therapy management. Additionally, health plans, pharmacies, and pharmacy benefit managers are beginning to partner on collaborative value-based pharmacy initiatives. Taken together, these efforts encourage a paradigm shift around drug cost management that more deeply integrates pharmacy into payment and delivery reform with the goal of improving quality and reducing the total cost of care.
\end{abstract}

KEY WORDS: payment reform; health policy; medication adherence; medication costs.

J Gen Intern Med 34(3):473-6

DOI: $10.1007 / \mathrm{s} 11606-018-4794-y$

(c) Society of General Internal Medicine 2019

$\mathrm{M}$ edications are one of the fastest growing sources of costs in the health system, ${ }^{1}$ and they are the cornerstone of chronic disease management. Despite extensive attention around drug pricing, medications have largely been a blind spot in initiatives that reform how healthcare is provided. For example, medication costs-particularly pharmacy-dispensed medications - have not been a meaningful component in

Prior presentations: This work has not been presented previously.

Received May 15, 2018

Revised October 15, 2018

Accepted December 6, 2018

Published online January 2, 2019 virtually any of the Centers for Medicare and Medicaid Services (CMS) value-based models, including the Comprehensive Primary Care (CPC) Initiative, Accountable Care Organizations (ACOs), and Bundled Payments for Care Improvement (BPCI) Initiative. This exclusion is in part because Medicare Part D (which covers prescription medication costs) is a separate and distinct benefit from Medicare Parts A and B (which cover hospital/facility-based and outpatient service costs, respectively) with its own regulatory and administrative requirements. ${ }^{2}$ Since Part D plan sponsors are private firms with clearly defined risks and responsibilities, the inclusion of Part $\mathrm{D}$ benefits in payment reform models has posed a challenge.

While medications are not explicitly part of their cost calculations, payment reform models may nevertheless influence medication costs and disease outcomes in varying ways. For example, payment reform models could lead to more efficient drug management with lower medication costs through increased use of generic medications and decreased use of costly, low-value medications. Conversely, they could lead to more efficient care management with higher medication adherence (particularly when reimbursement is linked to medication-related performance measures via pay-forperformance programs) and higher medication costs but reduced total healthcare costs due to reduced hospital admissions and emergency department visits. They alternatively could create incentives to use low-value medications in situations where doing so might reduce costs in the domains that are included in payment model calculations. For example, providers may perceive that giving unnecessary antibiotics for bronchitis or prescribing opioids for pain could prevent future office or emergency department visits.

What is the impact of payment reform models on medication use and costs? A synthesis of recent evidence from three prominent models - primary care-based redesign models, ACOs, and bundled payment programs-helps crystalize learnings from recent reforms about the effect of these models on medication use and guide future reforms that could incorporate medications in value-based models.

\section{PRIMARY CARE-BASED REDESIGN MODELS}

Primary care-based redesign models have been a central focus of both payment and delivery reform. The Patient-Centered 
Medical Home $(\mathrm{PCMH})$ model is a primary care-centric, practice-based transformation approach that emphasizes teambased care, enhancing patient-provider relationships, quality improvement, risk stratification, care coordination, and information technology. The primary form of additional payment for PCMHs is via risk-adjusted care coordination fees from payors. There is some evidence that structural components of primary care transformation in PCMHs improve adherence to therapy and reduce medication costs. A recent study assessing the association between PCMH accreditation and medication adherence found that patients managed in PCMHs exhibit up to 5\% greater adherence to chronic disease medications compared with those who are not. ${ }^{3}$ The CareFirst PCMH Program - which incorporated quality and utilization-based incentive payments for providers serving 1.4 million enrollees - was associated with a $2.8 \%$ reduction in total medical costs and $2.7 \%$ reduction in medication spending in 3 years. ${ }^{4}$

However, there is limited evidence to suggest that CMSsponsored primary care-focused payment models influence medication use or costs. The Centers for Medicare and Medicaid Innovation (CMMI) launched the CPC Initiative and subsequent $\mathrm{CPC}+$ models to test a better-resourced, accountable primary care-based model that emphasizes risk-stratified care management and coordination, performance-based incentive payments for quality and utilization metrics, and capital to invest in primary care practice transformation. Halfway through the 4-year program, the initiative improved quality but did not reduce medical costs. ${ }^{5}$ While comprehensive medication management was a key aspect of many practices' redesign strategies, medication costs were not included in total cost calculations so data are limited on how the CPC Initiative affected medication adherence and costs.

\section{ACCOUNTABLE CARE ORGANIZATIONS}

Accountable Care Organizations (ACOs) leverage a more comprehensive set of payment incentives that encompass accountability for total costs, thereby encouraging global redesign strategies for care improvement and cost reduction. Rather than addressing care at the practice level, they target a broader system of provider networks, focusing on aligning financial incentives and care coordination across providers to manage the health and healthcare costs of shared populations. Participating providers in ACOs may share in savings if the costs of their shared populations are below projected costs. Though many ACOs are composed of primary care groups, ACO models tend to be less prescriptive about requirements for primary care transformation and do not systematically provide up-front capital to support investment in primary care delivery.

Although medication adherence is a core component of quality metrics used to evaluate ACO performance, McWilliams et al. found that exposure to Medicare Shared Savings Program has not been associated with a meaningful improvement in the medication use and adherence among patients with cardiovascular disease and diabetes. ${ }^{6}$ Furthermore, Zhang's evaluation of Pioneer ACOs found that the medication use and costs did not substantially change despite a reduction in medical costs of $\$ 345$ per person per year. ${ }^{7}$ Though commercially sponsored ACOs have experimented with medications in total cost calculations, we are not aware of published evidence describing their outcomes and experiences.

\section{BUNDLED PAYMENT PROGRAMS}

Payment reform strategies that use bundled payments to promote efficiency and quality improvement aim to reduce variability and promote teamwork around episodes of care for specific procedures or conditions. Bundled payments apply fixed prices for certain episodes of care such as hip and knee joint replacements or coronary artery bypass grafting, and episodes generally include both acute and post-acute care costs. Hospitals may receive bonus payments if per-episode spending is less than fixed prices, or payback to payors if spending exceeds these target costs. Hospitals are, therefore, incentivized to coordinate care and manage costs across a continuum of an episode, and may financially align with non-hospital providers such as home health agencies and nursing facilities by sharing any bonuses received.

CMMI initiated their BPCI Initiative in 2013 to test four payment models for 48 clinical episodes. ${ }^{8}$ Notably, medication costs are absent from BPCI cost calculations, few medication-related measures are included as key quality measures, and the episodes are primarily focused on hospital-based rather than ambulatory care clinicians. In its first 21 months, evidence shows no change in quality and lower Medicare Parts A and B costs (largely from reduced post-acute spending), ${ }^{9}$ but effects on medication adherence and costs have not been systematically assessed likely due to their lack of emphasis in bundled payment models. At a regional level, Navathe et al. assessed changes in costs after BPCI implementation in the Baptist Health System. ${ }^{10}$ They found that average Medicare joint replacement expenditures declined by $20.8 \%$ between 2008 and 2015, while inpatient prescription drug costs declined by $2.4 \%$.

Although data are lacking on the impact of Medicare's BPCI Initiative on medication use and costs at the national level, there is some evidence on the impact of bundles on drug costs in the commercial setting nationally. In 2009, a national commercial insurer developed an oncology bundled payment program for patients with breast, lung, and colon cancer seen in five oncology practices. ${ }^{11}$ The bundle included both medical and medication costs, predominantly driven by physicianadministered medications. The program resulted in \$33.4 million in savings despite an increase in chemotherapy costs of $\$ 13.5$ million. While these models did little to address outpatient pharmacy utilization or costs, they highlight the potential to align pharmaceutical and medical cost-related incentives to drive better care at lower costs. 


\section{IMPLICATIONS}

The literature about the impact of payment and delivery reform models on medication adherence and costs could offer insights for policymakers, payors, and providers about how to best align incentives to positively influence outcomes while avoiding unnecessary medication costs. Although CMS has expressed interest in including medication costs in the total cost calculations, the structural exclusion of Part D medication costs from CMS-based alternative payment models, specifically CPC models, ACOs, and the BPCI Initiative, has complicated this goal. However, commercial models that integrate pharmacy and medical spending show promise and may serve as a guide for further model development.

Newer CMS models are underway that allow greater flexibility in pharmacy benefit design and that reward improved medication therapy management in Medicare Part D. For example, the Medicare Advantage Value-Based Insurance Design model allows for enrollees' cost sharing to be reduced for medications that treat select chronic diseases. ${ }^{12}$ Furthermore, the Enhanced Medication Therapy Management Model rewards Part D plans for improved medication adherence where plans that demonstrate cost savings receive premium subsidies in subsequent years to promote further enrollment. ${ }^{13}$

As we await feedback on their performance, there is a need to test broader integration of pharmacy-focused incentives in payment reform models, with greater alignment between health plans, pharmacies, and pharmacy benefit managers (PBMs) with providers around the total cost of care management. For instance, UPMC Health Plan now includes medication costs in its shared savings calculations for providers, and health plan-supported managed care pharmacists partner with the system's primary care physicians (PCPs) around opportunities to improve quality and reduce unnecessary costs associated with medications. Payment models that incorporate pharmacies could support deeper collaboration with providers to optimize medication choice and management. One such payment model is North Carolina's Community Pharmacy Enhanced Services Network, which aims to broaden care management and medication optimization services through care teams consisting of pharmacists, PCPs, care managers, and behavioral health providers. ${ }^{14}$ This model offers permember-per-month payments based on patient risk- and medication-related quality metric performance to community pharmacists who provide expanded services in PCMHs. These expanded services include electronic pharmacy care plan development, intensive medication reconciliation, adherence monitoring and coaching, and synchronization of chronic disease medication fill dates. Finally, payment models that incorporate PBMs could leverage more meaningful manufacturer discounts based on the impact on outcomes and total costs.

Taken together, these efforts can encourage an important paradigm shift around drug cost management from volumebased price negotiations to a true focus on value for patients and populations. Nevertheless, the exclusion of drug costs from payment reform model cost calculations leads to missed opportunities for health plans and health systems to prioritize effective medication use and management. In a healthcare landscape where payment reform models were developed in part to manage unsustainable healthcare costs, we cannot afford drugs to be a blind spot. With the continued growth of medications as a contributor to cost, quality, and value of care, their incorporation in payment reform models will be critical in order to enhance the potential of effective medication use in promoting high-value care.

Acknowledgments: Contributors: There were no other contributors to the manuscript.

Corresponding Author: Natasha Parekh, MD, MS; Division of General Internal Medicine, University of Pittsburgh, Lothrop Street, Pittsburgh, PA, USA (e-mail: nkp10@pitt.edu).

\section{Compliance with Ethical Standards:}

Conflict of Interest: Natasha Parekh is employed by the UPMC Center for High-Value Health Care and Center for Value-Based Pharmacy Initiatives. Mark McClellan is the Director of the Robert J. Margolis Center for Health Policy. William H. Shrank is the Chief Medical Officer of the UPMC Health Plan.

Publisher's Note: Springer Nature remains neutral with regard to jurisdictional claims in published maps and institutional affiliations.

\section{REFERENCES}

1. Altarum Institute Center for Sustainable Health Spending. Altarum Spending Brief \#17-8: June 2017 Data. Available at: https://altarum. org/sites/default/files/uploaded-related-files/CSHS-Price-Brief_Aug 2017.pdf. Accessed November 11th, 2018.

2. Medicare Payment Advisory Commission. Overview: Medicare Drug Spending. June 16, 2016. Available at: http://www.medpac.gov/docs/ default-source/fact-sheets/overview-of-medicare-drug-spending.pdf? sfvrsn=0. Accessed November 11th, 2018.

3. Lauffenburger JC, Shrank WH, Bitton A, et al. Association Between Patient-Centered Medical Homes and Adherence to Chronic Disease Medications: A Cohort Study. Ann Intern Med. 2017;166(2):81-88.

4. Cuellar A, Helmchen LA, Gimm G, et al. The Care First PatientCentered Medical Home Program: Cost and Utilization Effects in Its First Three Years. J Gen Intern Med. 2016;31(11):1382-1388.

5. Dale SB, Ghosh A, Peikes DN, et al. Two-Year Costs and Quality in the Comprehensive Primary Care Initiative. N Engl J Med. 2016;374(24):2345-56.

6. McWilliams JM, Najafzadeh M, Shrank WH, Polinski JM. Association of Changes in Medication Use and Adherence With Accountable Care Organization Exposure in Patients With Cardiovascular Disease or Diabetes. JAMA Cardiol. 2017;2(9):1019-1023.

7. Zhang Y, Caines KJ, Powers CA. Evaluating the Effects of Pioneer Accountable Care Organizations on Medicare Part D Drug Spending and Utilization. Med Care. 2017;55(5):470-475.

8. Centers for Medicare and Medicaid Services. Bundled Payments for Care Improvement (BPCI) Initiative: General Information. Updated October $25^{\text {th }}, 2018$. Available at: https://innovation.cms.gov/initiatives/bundled-payments/. Accessed November 11th, 2018.

9. Dummit LA, Kahvecioglu D, Marrufo G. Association Between Hospital Participation in a Medicare Bundled Payment Initiative and Payments and Quality Outcomes for Lower Extremity Joint Replacement Episodes. JAMA. 2016;316(12):1267-78.

10. Navathe A, et al. Cost of Joint Replacement Using Bundled Payment Models. JAMA Intern Med. 2017;177(2):214-222.

11. Newcomer LN, Gould B, Page RD. Changing physician incentives for affordable, quality cancer care: results of an episode payment model. J Oncol Pract. 2014;10(5):322-6. 
12. Centers for Medicare and Medicaid Services. Medicare Advantage Value-Based Insurance Design Model. Updated August 28 ${ }^{\text {th }}, 2018$. Available at: https://innovation.cms.gov/initiatives/vbid/. Accessed November 11th, 2018

13. Centers for Medicare and Medicaid Services. Part D Enhanced Medication Therapy Management Model. Updated October $23^{\text {rd }}, 2018$. Available at: https://innovation.cms.gov/initiatives/enhancedmtm/. Accessed November 11th, 2018.

14. Community Care of North Carolina. Community Pharmacy Enhanced Services Network. Available at: https://www.communitycarenc.org/ what-we-do/supporting-primary-care/pharmacy/cpesn. Accessed November 11th, 2018. 